\title{
Cognición situada y racionalidad. Hacia una ecología interactiva del razonamiento
}

\section{[Situated Cognition and Rationality. Towards an Interactive Ecology of Reasoning]}

\author{
ANA LAURA FONSECA PATRÓN \\ Universidad Autónoma de San Luis Potosí \\ alaurafon@gmail.com, ana.fonseca@uaslp.mx
}

\begin{abstract}
Resumen: La racionalidad ecológica que propone el grupo de investigación $\mathrm{ABC}$ destaca en su proyecto normativo la relación entre las heurísticas rápidas y frugales y el ambiente; por ello, considera que se trata de una racionalidad situada. El primer objetivo de este trabajo es mostrar que, si bien la racionalidad ecológica puede entenderse como una forma de situar la racionalidad, ello no implica situar el razonamiento. En particular, se muestra que la manera de entender la ecología del razonamiento es estática, como en los estudios estándar de la cognición. El segundo objetivo es mostrar que es deseable y viable caracterizar el razonamiento heurístico de manera que nos permita entender cómo las interacciones con el entorno son parte de las estructuras cognitivas que sustentan nuestras capacidades de razonamiento, es decir, formular una ecología interactiva del razonamiento.
\end{abstract}

Palabras clave: racionalidad acotada, racionalidad ecológica, razonamiento heurístico, cognición distribuida, prácticas

\begin{abstract}
The relation between fast and frugal heuristics and the environment is central to the normative project of the ecological rationality proposed by the $\mathrm{ABC}$ research group. This is why ecological rationality has been considered as a situated rationality. This paper shows that even if ecological rationality can be seen as situated rationality, this does not imply a situated form of reasoning and, in particular, that the understanding of the ecology of reasoning in this proposal is as static as it is in the standard studies of cognition. Next, the article focuses on showing that characterizing heuristic reasoning in a way that allows us to understand how interactions with the social and material environment are components of the cognitive structure that ground our reasoning is a desirable and viable task, which would allow us to formulate an interactive ecology of reasoning.

Key words: bounded rationality, ecological rationality, heuristic reasoning, distributed cognition, practices
\end{abstract}

\section{Introducción}

Este trabajo parte de la idea de que adoptar una perspectiva particular en los estudios situados sobre el razonamiento es relevante para la comprensión de la racionalidad. El llamado "movimiento de estudios 
situados de la cognición" engloba diversas propuestas de cómo comprender y estudiar la cognición de manera que sea posible entender los factores del ambiente físico, social y cultural que resultan relevantes para la adquisición, desarrollo y ejecución de diversas capacidades cognitivas (Smith 1999, pp. 769-770). A menudo, los estudios situados de la cognición se presentan como una solución potencial para algunos de los problemas más apremiantes en los estudios tradicionales de la cognición.

Una preocupación central en los estudios del razonamiento realizados desde mediados del siglo pasado en psicología cognitiva ha sido entender el papel que desempeñan las diferentes condiciones ambientales en la ejecución de problemas inferenciales específicos. Esto puede entenderse como el reconocimiento de que el razonamiento en general ha de estudiarse desde una perspectiva situada en el sentido de que, cuando los seres humanos razonamos normalmente, de una forma $\mathrm{u}$ otra, las características del contexto en el que nos encontramos influyen en el desarrollo del razonamiento. ${ }^{1}$ Una pregunta crucial para entender las implicaciones de esos estudios de la racionalidad humana es cómo dar cuenta de la ecología del razonamiento, es decir, cómo explicar la interacción entre el agente y el ambiente cuando aquél razona. ${ }^{2} \mathrm{El}$ interés de Herbert A. Simon por entender cómo con nuestras capacidades cognitivas - limitadas - podemos hacer frente a los problemas complejos que encontramos en el ambiente puede considerarse una manera de formular dicha pregunta. La idea detrás de su conocida metáfora de las tijeras (Simon 1990, p. 7) ${ }^{3}$ plantea la necesidad de estudiar la interacción entre el agente y su medio para entender la conducta inteligente. En tiempos más recientes, la propuesta de la racionalidad ecológica del grupo de investigación ABC (Adaptive Behavior and Cognition) incorpora como parte importante de su proyecto normativo la relación entre las heurísticas rápidas y frugales y el ambiente; por ello, el grupo considera que se trata de una racionalidad situada.

${ }^{1}$ Así, estudiar de forma situada el razonamiento no se refiere a un tipo de razonamiento, sino a una manera de caracterizarlo para su estudio.

${ }^{2}$ Aunque desde otra perspectiva, el señalamiento de Edwin Hutchins de entender la relevancia de lo que llama "la ecología de la cognición" responde a una preocupación similar. Para Hutchins, dicha ecología de la cognición se refiere a la comprensión del tipo de interacción entre el agente y el medio durante la ejecución de ciertas capacidades cognitivas (Hutchins 2010).

${ }^{3}$ Para Simon, así como una tijera sólo puede cortar cuando interactúan sus dos hojas, el comportamiento inteligente sólo puede suceder en la intersección entre el medio y las capacidades cognitivas de los agentes (Simon 1990, p. 7).

Revista de Filosofía Diánoia, vol. 64, no. 83 (noviembre de 2019-abril de 2020) e-ISSN: 1870-4913 • DOI: https://doi.org/10.22201/iifs.18704913e.2019.83.1618 
El primer objetivo de este trabajo es mostrar que, si bien la racionalidad ecológica puede entenderse como una manera de situar la racionalidad, ello no implica un estudio situado del razonamiento y presenta una caracterización de la ecología del razonamiento estática, como en los estudios estándares de la cognición. El segundo objetivo es mostrar que es deseable y viable caracterizar el razonamiento heurístico de manera que nos permita entender cómo las interacciones con el entorno son parte de las estructuras cognitivas que sustentan nuestras capacidades de razonamiento, es decir, formular una ecología interactiva del razonamiento.

En primer lugar, expondré la manera en que la propuesta de la racionalidad ecológica que desarrollan Gerd Gigerenzer y su grupo de investigación $\mathrm{ABC}$ se considera afín a los estudios de cognición situada. Después, mostraré que en dicha propuesta se conservan algunos de los supuestos principales de la cognición estándar, lo que da lugar a una ecología del razonamiento estática que dificulta explicar lo que Simon llamó "problemas mal estructurados" y también obstaculiza dar cuenta de cómo generamos y modificamos nuestros patrones de decisión a través de la experiencia. Por último, en la tercera sección mostraré apelando a estudios empíricos que es posible y deseable entender la ecología del razonamiento de una manera interactiva, es decir, de una forma que nos permita entender cómo generamos patrones estables para responder al ambiente a través de la interacción del agente con su ambiente social y material. La idea que subyace en este artículo es que la relevancia de estudiar el razonamiento de forma situada podría conducirnos a una mejor comprensión de la racionalidad humana, es decir, a una comprensión naturalizada — sin dejar de tomar en cuenta las limitaciones y potencialidades de la cognición humana-. La tesis es que contar con una comprensión de cómo está situado nuestro razonamiento nos ofrece una mejor descripción de éste que una teoría que haga abstracción de los factores del contexto. Si bien ahora no diré mucho acerca cómo sería en concreto esa dimensión normativa, me centraré en un paso que considero previo, a saber, mostrar que es deseable y viable contar con una ecología del razonamiento interactiva. La racionalidad ecológica que proponen Gigerenzer y sus colegas reconoce la necesidad de formular una caracterización más rica del razonamiento; de lo que se trata es de analizar el alcance de la incorporación de los factores del contexto en dicha propuesta. 


\section{Racionalidad situada de $A B C$}

La propuesta de la racionalidad ecológica del grupo $\mathrm{ABC}$ afirma que gran parte de las decisiones humanas se encuentran guiadas por heurísticas de decisión comúnmente simples que aprovechan la estructura del ambiente para llevar a cabo buenas elecciones. Se trata de un enfoque situado al estudio de la racionalidad humana en cuanto que otorga un papel crucial a la manera en que los individuos apelan a propiedades estructurales del ambiente para tomar decisiones adaptativas. Gigerenzer retoma la metáfora de las tijeras de Simon para explicar su proyecto, el cual, de hecho, considera una continuación de la investigación sobre la racionalidad acotada que comenzó Simon, pero con un énfasis mayor en el papel del ambiente (Gigerenzer y Todd 1999, p. 13). Simon discutió en forma abierta con proponentes de la acción situada (Vera y Simon 1993a; Vera y Simon 1993b); sin embargo, a pesar de que Gigerenzer considera su propuesta de racionalidad ecológica como heredera de la racionalidad acotada, él y otros defensores de la racionalidad ecológica del grupo $A B C$ no tienen problema en afirmar que su propuesta es afín a los estudios de la cognición situada. ${ }^{4}$ Así, Henry Brighton y Peter Todd en su artículo "Situating Rationality" (Brighton y Todd 2009) repasan el sentido en el que se entienden en su propuesta de racionalidad ecológica cada una de las seis características que Brian Cantwell Smith identificó como distintivas de los estudios situados de la cognición en el artículo "Situatedness/Embeddedness" de la MIT Encyclopedia of Cognitive Sciences, a saber, que la cognición es social, corporeizada, concreta, localizada, involucrada en interacciones con el ambiente y específica (Smith 1999).

Uno de los principales objetivos del proyecto del grupo ABC es proporcionar una teoría de la racionalidad humana que sea psicológicamente aceptable o descriptivamente adecuada. Para Brighton y Todd la propuesta de racionalidad ecológica, más que ser resultado del análisis filosófico acerca del razonamiento correcto - como en la teoría tradicional o estándar de la racionalidad $^{5}$ a la que el grupo $\mathrm{ABC}$ se

\footnotetext{
${ }^{4}$ Una de las afirmaciones más importantes de los estudios de la acción situada es que los humanos y sus interacciones con el mundo no pueden entenderse con modelos de sistemas físicos de símbolos, como pretendía Simon, sino a través de la observación de la conducta en contextos reales (Vera y Simon 1993a). Así, aunque podamos reconocer que los estudios de la acción situada no son estrictamente el mismo movimiento que el de los estudios de la cognición situada, está claro que comparten el objetivo de estudiar la cognición de forma contextual.

${ }^{5}$ Por lo común, "visión estándar de la racionalidad" se entiende como los cri-
} 
opone y critica (Gigerenzer y Todd 1999, pp. 5-12; Brighton y Todd 2009, pp. 332-336)—, es resultado de la investigación empírica de la cognición. Las afirmaciones normativas en esta propuesta se derivan de las observaciones de estudios empíricos (Gigerenzer y Sturm 2012, p. 244). Así, una primera observación que vale la pena hacer es que Brighton y Todd parecen no distinguir de una manera clara entre las características de la cognición y los rasgos de la propuesta normativa del equipo $\mathrm{ABC}$. Los autores no hablan de una "cognición situada" sino de la "racionalidad situada" y contrastan las características que enuncia Smith con su caracterización de la racionalidad, no con su caracterización del razonamiento. Como se verá, esto es relevante, pues, en el fondo, los integrantes de $\mathrm{ABC}$ no cuestionan ni la caracterización de la cognición como algo que sucede completamente en el cerebro ni del ambiente como un repositorio de información que, si bien es importante comprender, desempeña un papel pasivo en nuestros procesos de razonamiento. Las formas en que siguen las seis características enunciadas por Smith son las siguientes:

1) La racionalidad ecológica está localizada. Una heurística es racional según la propuesta de los integrantes de $A B C$ cuando permite resolver con éxito un tipo de tarea en un ambiente particular. Sin el ambiente no hay manera de saber si una heurística es racional o no; por lo tanto, consideran que su propuesta está localizada (Brighton y Todd 2009, p. 339; Gigerenzer y Sturm 2012, p. 246). El ambiente para el proyecto $A B C$ significa la estructura informacional del ambiente (Gigerenzer 2001, p. 39). Esta manera de entender el papel del ambiente en la racionalidad tiene su origen en la ya mencionada metáfora de las tijeras de Simon. Que la racionalidad ecológica esté localizada puede entenderse como la importancia que $\mathrm{ABC}$ concede a la hoja del ambiente en dicha metáfora.

2) La racionalidad ecológica es concreta. Así como la característica de la localización se asocia a la hoja del ambiente en la metáfora de Simon, para el grupo ABC entender la cognición como algo concreto se asocia a la hoja de las capacidades cognitivas en la misma metáfora. La propuesta de racionalidad ecológica del grupo $\mathrm{ABC}$ se concibe como concreta en dos grados: en un nivel puramente computacional, que tiene que ver con la limitación general

terios de buen razonamiento de las reglas de la lógica deductiva, el cálculo de probabilidades y las teorías formales de la decisión (Stein 1996).

Revista de Filosofía Diánoia, vol. 64, no. 83 (noviembre de 2019-abril de 2020) e-ISSN: 1870-4913 • DOI: https://doi.org/10.22201/iifs.18704913e.2019.83.1618 
—en los seres humanos y en las máquinas- de procesar cierta cantidad de información y, también, en un sentido biológico, que nos habla de las limitaciones de procesamiento de información propias de los seres humanos; por ejemplo, identificar formas de representación informacional que nos son más fácilmente procesables o accesibles. Lo relevante de tomar en cuenta las restricciones cognitivas para el grupo $\mathrm{ABC}$ es que algunas de ellas permiten aprovechar con éxito el ambiente (Brighton y Todd 2009, p. 339).

3) La racionalidad ecológica está involucrada con el ambiente. En la racionalidad ecológica las heurísticas son especializadas, pues funcionan en un ambiente particular para resolver tareas bien definidas. Casi cada heurística propuesta por el equipo de $\mathrm{ABC}$ puede descomponerse en tres reglas de inferencia: una de búsqueda de información, una de detención de la búsqueda y una de decisión (Marsh, Todd y Gigerenzer 2004, p. 275). El grupo considera que toda heurística que cuenta con una regla de búsqueda tiene la característica de estar involucrada con el ambiente porque, durante el proceso de búsqueda de información, el sujeto está implicado en una interacción con el ambiente y percibe la tarea inferencial que realiza como una tarea en curso y no como una actividad estática (Brighton y Todd 2009, p. 339). En la próxima sección analizaré con cierto detalle cómo se entiende esa interacción con el ambiente.

4) La racionalidad ecológica es específica. Esto se debe a que la especificidad de dominio de cada heurística intenta captar distintas contingencias en las tareas que la gente enfrenta. Por ejemplo, cuando los agentes tienen que actuar en un tiempo limitado la elección de la estrategia de decisión cambia por esa limitante. En este caso, contar con una presión de tiempo es una contingencia en la tarea que se enfrenta. La argumentación del proyecto $A B C$ sostiene que las heurísticas pueden responder bien a factores ambientales contingentes como éstos; en este caso particular, querría decir que algunas heurísticas funcionan mejor cuando existe una limitación de tiempo en los agentes que cuando ésta no existe. Juzgar si el uso de una heurística u otra es adecuado depende de los factores o las contingencias de cada tarea; por lo tanto, sólo podemos hablar normativamente de las heurísticas en relación con una situación particular (Brighton y Todd 2009, p. 340). 
5) La racionalidad ecológica está corporeizada. En el caso de los investigadores de $\mathrm{ABC}$, la corporeización es importante en la medida en que los agentes con un diseño morfológico distinto resolverán problemas mediante procesos distintos. Éste es un sentido muy claro en el que la cognición depende de la concreción física particular del agente cognitivo. Sin embargo, es una manera muy limitada en la que los aspectos corpóreos de los agentes entran en juego para el desarrollo cognitivo. Algunos estudios de cognición situada toman esta característica en un sentido distinto, según el cual la corporeidad de los sujetos tiene un papel importante en el despliegue de las capacidades cognitivas; por ejemplo, cuando se observa que al llevar a cabo tareas cognitivas descargamos parte del esfuerzo cognitivo en acciones motoras (Kirsh y Maglio 1994). ${ }^{6}$ Para los investigadores del grupo ABC la corporeidad del agente forma parte de sus capacidades cognitivas en cuanto que la cognición está restringida a aquello que podemos o no percibir y hacer. La manera de entender la corporeización de la cognición en el proyecto de $\mathrm{ABC}$ tiene que ver con la aceptación de una distinción clara entre cognición de orden superior y cognición de orden inferior. Las heurísticas cognitivas se consideran parte de la cognición de orden superior y dependen de la cognición de orden inferior (percepción y, en general, la corporeización) sólo en la medida en que se nutren de sus insumos (Brighton y Todd 2009, p. 340).

6) La racionalidad ecológica es social. Difícilmente algún tipo de estudio de la cognición negaría que ésta sucede en un ambiente social; sin embargo, la manera exacta en que los factores sociales inciden o forman parte de la cognición puede concebirse en diferentes sentidos. Los aspectos sociales forman parte del proyecto de racionalidad ecológica en la medida en que se reconoce que "una parte significativa de la estructura del ambiente a menudo estará constituida por otros individuos y el resultado de sus acciones" (Brighton y Todd 2009, pp. 340-341); cuando esto sucede se considera un caso especial de la racionalidad ecológica. En el grupo $\mathrm{ABC}$ se distinguen heurísticas de diferentes tipos; entre ellas, las heurísticas perceptivas, las cognitivas y las sociales (Gigerenzer

\footnotetext{
${ }^{6}$ También hay estudios que revelan que incluso en las actividades que en lo inmediato aparentemente no requieren el cuerpo para ser llevadas a cabo (como planear alguna actividad) se activan las zonas sensomotrices del cerebro. Esto indica que esas actividades tienen una dependencia de las funciones motoras del cerebro (Robins y Aydede 2009a, p. 5)
}

Revista de Filosofía Diánoia, vol. 64, no. 83 (noviembre de 2019-abril de 2020) e-ISSN: 1870-4913 • DOI: https://doi.org/10.22201/iifs.18704913e.2019.83.1618 
y Todd 1999, pp. 29-31). Las sociales son las que lidian con el caso especial de la racionalidad ecológica en ambientes sociales (Brighton y Todd 2009, p. 341). Aunque se podría cuestionar la claridad de la distinción, para este texto lo relevante es señalar que, a pesar de que el grupo $\mathrm{ABC}$ reconoce cierto papel de lo social en la conformación de algunas heurísticas, la racionalidad ecológica no es social; es decir, aquello que nos dice si una heurística es exitosa y, por lo tanto, normativa en un ambiente específico, no tiene que ver con los aspectos sociales de la cognición, sino con la precisión, la rapidez y la economía de los recursos con los cuales ésta permite resolver un problema determinado.

\section{La ecología del razonamiento en la racionalidad ecológica del grupo $A B C$}

El grupo $A B C$ considera de manera explícita que su propuesta de racionalidad ecológica es afín al movimiento de estudios situados de la cognición. En la sección anterior presenté el sentido que toman algunas de las características típicamente asociadas a ese movimiento en la racionalidad situada que proponen. En esta sección explicito algunas características de la ecología del razonamiento implícita en dicha propuesta y hago ver que la relación entre el agente y su medio se concibe a partir de cortes discretos que ofrecen una visión estática del proceso de razonamiento, lo que dificulta la comprensión de cómo la experiencia conduce a modificaciones en la conducta y al desarrollo de nuevas estrategias. Para facilitar ese análisis conviene tener en cuenta como ejemplo una de las heurísticas propuestas por el ABC: "Toma lo mejor". Esta heurística permite resolver problemas que consisten en elegir uno de dos objetos de acuerdo con cierto criterio cuando se conoce al menos cierta información de cada uno de ellos; por ejemplo, elegir cuál de dos ciudades de las que se ha escuchado hablar antes tiene más población. Las reglas de inferencia que forman la heurística son: 1) escoger la pista que tenga la mayor validez (el fragmento de información que se considere que tenga una mayor relación con el objetivo del problema); 2) observar si alguna de las dos opciones entre las que hay que elegir cumple con la pista de la mayor validez. Si sólo una de ellas cumple con esa pista, detener la búsqueda, y 3) elegir la opción que la cumple. Si ambas opciones o ninguna de ellas cumple con la pista de mayor validez, repetir el procedimiento con la pista que siga en validez. Uno de los problemas con los que suele presentarse la heurística es elegir cuál de dos ciudades tiene mayor población, un problema que se puede considerar cotidiano (Gigerenzer y Goldstein 1999, pp. 84-85). Las pistas 
podrían ser: si la ciudad tiene una universidad pública reconocida, si tiene un equipo de futbol en primera división, etcétera.

Características de la ecología del razonamiento del grupo $A B C$

a) El ambiente

El proyecto de racionalidad ecológica pretende encontrar patrones de decisión empleados en las actividades de la vida diaria, y ciertamente varios de sus modelos de heurística se explican como formas de tomar decisiones que pueden considerarse cotidianas. El procedimiento que sigue el grupo para encontrar heurísticas es diseñar modelos computacionales que se consideren candidatos a ser heurísticas simples en nuestra caja de herramientas adaptativas; una vez diseñados esos modelos computacionales, se evalúa su ejecución en diferentes estructuras informacionales también incorporadas en algún programa computacional. Un primer paso para entender la relación entre el agente y su ambiente en la racionalidad ecológica es especificar el significado de "ambiente" y, aunque puede ser materia de otro artículo, por ahora lo importante aquí es señalar que es un término que puede resultar ambiguo porque algunas veces parecería que se emplea en el sentido de aquello que nos rodea en términos generales, social, natural y materialmente; sin embargo, esta acepción de ambiente se niega de manera explícita y se afirma que "[E]l término ambiente, aquí, no se refiere a una descripción del ambiente físico y biológico total, sino sólo a esa parte importante para el organismo dadas sus necesidades y objetivos" (Gigerenzer 2001, p. 39). Una posible interpretación de esta cita es que se refiere al ambiente físico en el que habitamos pero sin tomarlo en su conjunto, sino sólo de acuerdo con las características que plantean algún problema o algún beneficio para determinados organismos. Sin embargo, cuando se trata de describir los ambientes en los cuales las heurísticas son exitosas, está claro que se refiere más bien a la estructura informacional, es decir, a ciertos fragmentos de información que son insumos para cierta regla de inferencia (la heurística). Así, Gigerenzer y Brighton afirman:

[U]n ambiente es una colección de objetos. Cada objeto relaciona $m$ pistas binarias con un criterio entero. Las dos clases de ambiente consideradas aquí están parametrizadas por $m$. La primera clase de ambientes comprende ambientes binarios, cada uno de los cuales tiene $2^{m}$ objetos definidos por:

Revista de Filosofía Diánoia, vol. 64, no. 83 (noviembre de 2019-abril de 2020) e-ISSN: 1870-4913 • DOI: https://doi.org/10.22201/iifs.18704913e.2019.83.1618 


$$
H_{\text {binario }}(m)=\left\{\left\langle b_{m}(i), i\right\rangle: 0 \leq i \leq\left(2^{m}-1\right)\right\}
$$

donde la función $b_{m}(i)$ mapea enteros en sus representaciones binarias, codificados usando las pistas binarias (por ejemplo, $b_{4}(3)=(0,0,1,1)$ ). Los ambientes binarios tienen pesos no compensatorios y las pistas no están correlacionadas. (Gigerenzer y Brighton 2009, p. 142)

Esto quiere decir que el ambiente se restringe a unos cuantos objetos que están relacionados en cierta forma con variables específicas. La noción de ambiente que resulta relevante para su propuesta tanto descriptiva como normativa y de diseño de las heurísticas es esta noción de ambiente como estructura informacional. ${ }^{7}$ Una manera de armonizar estos dos sentidos es suponer que la segunda acepción —el ambiente como estructura informacional- de alguna manera capta adecuadamente todas las características presentes en nuestro entorno que nos son relevantes al momento de tomar decisiones y resolver problemas de un tipo particular. Este supuesto está presente en la propuesta de la racionalidad ecológica, pues esa segunda acepción es todo lo que se necesita para dar cuenta de la racionalidad situada de sus heurísticas rápidas y frugales.

En el caso de la heurística "Toma lo mejor" que elegí como ejemplo, el éxito se puede esperar cuando cada uno de los objetos en cuestión tiene ciertos valores o pistas que están relacionados en mayor o menor medida con el criterio de elección y algunas de esas pistas, pero no todas, son desconocidas para el agente (Marsh, Todd y Gigerenzer 2004, p. 278). Estas circunstancias específicas en que la heurística sería exitosa constituyen el ambiente en el que deberíamos emplearla. Esta noción de ambiente puede referirse a muy diversos problemas, como elegir entre universidades en las cuales estudiar, empresas en las cuales invertir, coches que comprar, etc. A través de esta heurística podemos ver que la propuesta de heurísticas rápidas y frugales no toma en cuenta quién ni cuándo se toma la decisión o se resuelve un problema; lo que importa es que una vez reconocida cierta estructura ambiental, se aplique la heurística adecuada.

\footnotetext{
${ }^{7}$ Por supuesto, el problema no es que la caracterización del ambiente se modele formalmente. El señalamiento apunta a mostrar qué es lo que, dada la manera en que se formaliza la noción de ambiente, resulta relevante para su caracterización en la propuesta de $\mathrm{ABC}$.
}

Revista de Filosofía Diánoia, vol. 64, no. 83 (noviembre de 2019-abril de 2020) e-ISSN: 1870-4913 • DOI: https://doi.org/10.22201/iifs.18704913e.2019.83.1618 
b) El agente

El otro elemento en la relación ecológica es, por supuesto, el agente. En este caso, el agente se caracteriza a través de las heurísticas rápidas y frugales de la caja de herramientas adaptativas. Los sentidos - como mencioné antes, al enunciar la característica de la corporeización de la racionalidad situada - sólo proveen insumos para las heurísticas, pero para explicar la racionalidad basta con apelar a las reglas de inferencia simples que constituyen las heurísticas, lo que el equipo de ABC llama "los bloques de construcción" de una heurística. Por lo común, se trata de tres reglas sencillas, una de búsqueda de la información, una de detención de la búsqueda y otra de decisión, a las cuales corresponden respectivamente las reglas expuestas al presentar como ejemplo la heurística "Toma lo mejor". Así, la relación entre las heurísticas y el ambiente es la relación entre reglas de inferencia simples (el agente) con ciertos fragmentos de información (el ambiente) con las que aquéllas pueden funcionar. Una vez que se han encontrado las reglas de inferencia que forman una heurística, la implementación material que ésta tenga deja de ser importante. Eso es lo que permite a los investigadores de $A B C$ hacer que las pruebas de si una heurística es exitosa en cierto ambiente (estructura informacional) puedan realizarse mediante simulaciones computacionales. Para la racionalidad ecológica, el agente se restringe a las reglas de inferencia que se encuentran dentro de su caja de herramientas adaptativas.

En el caso de la heurística "Toma lo mejor" las tres reglas de inferencia mencionadas (elegir las pistas con mayor validez, observar cuál opción cumple con las pistas y elegir la opción que cumpla con alguna de las pistas cuando la otra opción no la cumple) son todo lo que cuenta como "el agente", pues se considera que estas reglas de inferencia sencillas forman parte del aprovisionamiento biológico de los seres humanos, es decir, forman parte de la caja de herramientas adaptativas. Entonces, el ambiente en el cual se aplica la heurística se entiende como las ciudades de las que se habla en el problema y las respectivas pistas o fragmentos de información que se tengan de ellas, mientras que el agente se refiere a las reglas de inferencia que se aplican para buscar la información, detener la búsqueda de la información y tomar la decisión.

c) La relación entre el agente y el medio

Para entender mejor la interacción entre el agente y su ambiente en la racionalidad ecológica, conviene recordar una distinción que Herbert Simon trazó entre los problemas bien estructurados y los problemas 
mal estructurados. Los problemas bien estructurados son aquellos que pueden formularse explícita, cuantitativamente, y que pueden resolverse mediante técnicas computacionales, mientras que los mal estructurados son aquellos que no cumplen con al menos una de las tres características anteriores (Simon 1958, pp. 4-5). Para Simon, la mayoría de nuestros problemas cotidianos son problemas mal estructurados debido a los grados de incertidumbre del ambiente y a las características propias de la cognición humana (Simon 1990, p. 6). Una estrategia para tratar de comprender la solución de los problemas mal estructurados es buscar su transformación en problemas bien estructurados. Podemos reconocer este tipo de estrategia en el proyecto de $A B C$ al advertir que su propuesta implica abstraer ciertas condiciones ambientales (quedándose sólo con lo que se entiende como la estructura en que se presenta la información disponible) hasta encontrar una heurística (regla de inferencia básica) con la cual los casos que caen en esas condiciones se puedan resolver (Marsh, Todd y Gigerenzer 2004, p. 276).

Esto supone que el razonamiento - caracterizado como la toma de decisiones y la resolución de problemas - se considera independiente de las interacciones sensoriales y motoras del agente con el mundo físico en la misma forma en que éstas eran irrelevantes en propuestas cognitivistas clásicas como la de Simon. La idea que deseo destacar aquí es que la racionalidad ecológica se presenta como una propuesta afín a los estudios situados del razonamiento y, como vimos en la primera sección, afirma que su propuesta está corporizada; sin embargo, la relevancia y el tipo de consideración que tienen del cuerpo del agente y su percepción es básicamente la misma que en los estudios tradicionales o clásicos — no situados- de la cognición. En el caso de la racionalidad ecológica, no hay una retroalimentación o un intercambio constante entre el sujeto y el ambiente. Una vez que hay ciertos insumos, se aplica una estrategia y se obtienen los resultados; el análisis de las heurísticas en cuanto que se consideran procesos cognitivos se detiene ahí. De manera que las heurísticas rápidas y frugales que propone el equipo $\mathrm{ABC}$ siguen siendo estrategias de decisión para problemas abstractos, bien estructurados, que pueden aplicarse en casos cotidianos de muy diverso contenido y que están restringidos por la caracterización que se hace de la estructura de la tarea por resolver. Así, si en lugar de considerar el problema de elegir entre cuál de dos ciudades tiene mayor población estuviéramos frente al problema de elegir entre cuál de dos seguros contratar, la estrategia sería la misma siempre que tuviéramos al menos cierto conocimiento de ambos seguros. La manera en que se establece una relación entre el agente y la resolución de una tarea

Revista de Filosofía Diánoia, vol. 64, no. 83 (noviembre de 2019-abril de 2020) e-ISSN: 1870-4913 • DOI: https://doi.org/10.22201/iifs.18704913e.2019.83.1618 
mediante la búsqueda de una regla de inferencia en la visión estándar de la racionalidad no es, entonces, muy distinta del proceso que propone Gigerenzer. Lo que es distinto es aquello que se considera normativo.

d) La normatividad

Llegamos así al cuarto elemento importante en la racionalidad ecológica, a saber, la normatividad. Una heurística se considera normativa en dos sentidos: cuando resulta exitosa para resolver una tarea al menos en un buen número de casos y cuando resulta más exitosa que otras estrategias de decisión, por ejemplo, que las estrategias optimizadoras (Gigerenzer y Goldstein 1999, pp. 82-95). La evaluación de si las heurísticas rápidas y frugales que proponen son más exitosas o no que las estrategias de decisión optimizadoras en la solución de algún tipo de problema se da mediante una simulación computacional de la rapidez, frugalidad y precisión con que cada una de las estrategias en cuestión permite resolver cierto problema (Gigerenzer y Goldstein 1999, pp. 75-95). Así, el estudio de las relaciones entre el agente y su medio - la ecología del razonamiento - se lleva a cabo mediante una abstracción de las actividades cotidianas similar a la que se emplea en los estudios estándar de la cognición, a los que suelen oponerse los estudios situados.

Cuando en $\mathrm{ABC}$ se habla de que la racionalidad ecológica es una manera de entender que la racionalidad está situada se hace referencia al estudio situado de la cognición de orden superior. ${ }^{8}$ Cómo sucede la interacción entre el medio y la cognición de orden inferior es una pregunta que la propuesta del grupo ABC no intenta responder. Se buscan las estrategias cognitivas y se trata de entender cómo esas estrategias que se encuentran dentro del cerebro responden ante información del exterior que, de alguna manera, ya ha sido codificada en cierto formato representacional con el fin de que pueda utilizarse para seguir las reglas de inferencia especificadas en las heurísticas que proponen. Cómo ha sucedido esa codificación es algo que no se considera relevante para entender las heurísticas del razonamiento.

\footnotetext{
${ }^{8}$ En la concepción de la cognición clásica fodoriana las funciones cognitivas superiores o cognición superior se asocian con la toma de decisiones, la resolución de problemas y, en general, con el razonamiento. En la propuesta original de Fodor estas funciones cognitivas se realizan en el procesador central, mientras que las funciones cognitivas inferiores se llevan a cabo en los módulos periféricos y proveen insumos para la realización de las funciones superiores. La idea aquí no es que Gigerenzer y sus colegas se comprometen con la visión fodoriana clásica de la cognición, pero sí que adoptan en forma una distinción de ese tipo.
}

Revista de Filosofía Diánoia, vol. 64, no. 83 (noviembre de 2019-abril de 2020) e-ISSN: 1870-4913 • DOI: https://doi.org/10.22201/iifs.18704913e.2019.83.1618 
En la propuesta que examino la cognición de orden superior - a la cual se asocian las heurísticas del razonamiento- se puede estudiar de forma independiente y autónoma de la cognición de orden inferior; en consecuencia, la normatividad de las heurísticas rápidas y frugales que proponen sólo depende de que la estrategia sea exitosa para resolver una tarea específica y que lo sea de forma casi tan precisa y más rápida y más frugal que otras estrategias más complejas. Es decir, se trata de una competencia entre algoritmos donde lo importante es cuál algoritmo es más rápido y frugal sin dejar de conservar grados aceptables de precisión en sus respuestas. En este sentido, la racionalidad ecológica que propone el equipo $\mathrm{ABC}$ está situada respecto de una estructura informacional y de otras estrategias de decisión y de solución de problemas.

Para que una heurística rápida y frugal se considere normativa para un tipo de tarea particular, su estructura inferencial debe permitir resolver un problema cuya información tiene cierta estructura específica. La heurística "Toma lo mejor" que he tomado como ejemplo es normativa no sólo porque sea exitosa para resolver los problemas con la estructura informacional señalada, sino también porque su tasa de éxito en la solución de ese tipo de tareas en comparación con las estrategias de decisión optimizadoras es alta (Gigerenzer y Goldstein 1999, pp. 8991). El análisis del éxito de las heurísticas y de su normatividad no toma en cuenta otra interacción entre el agente y su ambiente más que la que se da entre las reglas de inferencia que constituyen la heurística y la estructura informacional del ambiente a las que éstas responden. Lo que quiero señalar es que esta manera de entender la racionalidad es en cierto sentido similar a la manera en que se entiende la visión estándar de la racionalidad que Gigerenzer y sus colegas critican. En ambos casos se requiere identificar cierta estructura en un problema y la estrategia (o regla de inferencia) que permite resolverlo.

\section{Racionalidad situada sin razonamiento situado}

La caracterización del ambiente, del agente, de la relación entre ellos y de la normatividad de las heurísticas nos ofrece una imagen de la ecología del razonamiento discreta y estática. Se trata de una imagen discreta de la relación entre agente y ambiente porque podemos atomizar "episodios" de razonamiento heurístico en una estrategia inferencial especificable en pasos bien definidos (la heurística). Es una relación estática porque no da cuenta de la generación ni de la transformación de los procesos de razonamiento que tienen lugar mediante la interacción

Revista de Filosofía Diánoia, vol. 64, no. 83 (noviembre de 2019-abril de 2020) e-ISSN: 1870-4913 • DOI: https://doi.org/10.22201/iifs.18704913e.2019.83.1618 
continua entre el agente y su medio. Desde esta manera de entender la relación entre el agente y su medio es difícil explicar cómo la experiencia en el uso de ciertas estrategias puede dar lugar a modificaciones de las estrategias en uso y a la generación de algunas nuevas. ${ }^{9}$ No obstante, ésta es una característica distintiva del razonamiento heurístico que lo hace relevante para la comprensión de nuestro razonamiento en general. En resumen, aunque la racionalidad ecológica que propone Gigerenzer enfatice el papel del ambiente en el estudio del razonamiento heurístico, no se aparta de la concepción de la cognición que tenía Simon ni de cómo debemos caracterizar la relación entre el agente y su medio. En este sentido, la propuesta de racionalidad ecológica no se contrapone ni se aleja mucho de los estudios corrientes de la cognición. Por ello, no es trivial que Brighton y Todd hablen de racionalidad situada y no de cognición situada. Las heurísticas, tal como las modelan Gigerenzer y sus colegas, se aplican en problemas bien estructurados (en los que sabemos que hay una respuesta, como en el caso de qué ciudad es más grande, en nuestro ejemplo) o a problemas mal estructurados que, en cierta medida, se transforman en problemas bien estructurados (como elegir qué carrera estudiar o si debemos cambiar de trabajo). La idea central hasta aquí ha sido mostrar que el énfasis de Gigerenzer en la importancia del ambiente para entender la racionalidad humana va en la dirección correcta, pero no está acompañado de una caracterización de la cognición que explique en forma satisfactoria cómo la interacción con el medio forma parte de nuestros procesos de razonamiento al sostener una visión computacionalista à la Simon.

El componente ecológico en la propuesta del grupo $\mathrm{ABC}$ reside en la manera de evaluar el razonamiento heurístico (modelado a través de heurísticas rápidas y frugales), y por ello hablan de una racionalidad situada pero no hay propiamente un estudio de cómo está situado el razonamiento. El razonamiento se caracteriza como un conjunto de reglas de inferencia simples susceptibles de modelaje computacional; el único papel de los sentidos es proveer insumos para esas reglas básicas. Podemos decir que Gigerenzer ofrece una ecología de la racionalidad sin una ecología del razonamiento.

\section{Hacia una ecología interactiva del razonamiento}

Como hemos visto, en la racionalidad ecológica de Gigerenzer persiste la idea tradicional de que la cognición sucede y se explica principal-

${ }^{9}$ Una crítica de este tipo puede encontrarse en Bardone 2011.

Revista de Filosofía Diánoia, vol. 64, no. 83 (noviembre de 2019-abril de 2020) e-ISSN: 1870-4913 • DOI: https://doi.org/10.22201/iifs.18704913e.2019.83.1618 
mente a partir de lo que tiene lugar dentro del cerebro, como suponía Simon. El supuesto de que la cognición es aquello que pasa dentro del cerebro restringe las maneras en que podemos caracterizar la relación entre el agente (en el caso particular del razonamiento reducido a ciertos modelos de inferencia, como las reglas de inferencia de los cálculos lógicos o las heurísticas rápidas y frugales que propone Gigerenzer) y su medio (las estructuras informacionales que están fuera de él). En este tipo de relación el cerebro es la parte activa, como se puede ver en el caso de la heurística "Toma lo mejor", mientras que el ambiente es una parte pasiva, un repositorio de información que restringe o potencia (según sea el caso) nuestras capacidades cognitivas. ${ }^{10}$ Así, Peter Todd reconoce que entre los temas por resolver en la propuesta de la racionalidad ecológica se encuentra entender por qué elegimos las heurísticas que elegimos, cómo las obtenemos, cómo generamos nuevas heurísticas (Todd 2001, pp. 67-69). ${ }^{11}$ Resolver estas preguntas tiene que ver con entender la manera en que percibimos cierta situación que enfrentamos no sólo en términos aislados de aquello que en un momento preciso observamos, sino a partir de aquello que hemos experimentado en el pasado y que nos permite generar una respuesta adecuada o inadecuada.

Una ecología del razonamiento apuntaría a entender cómo se generan los patrones o modos estables de responder al ambiente a través de la interacción social y de la interacción del agente con su ambiente material. En la medida en que la racionalidad ecológica plantea que las heurísticas rápidas y frugales son resultado de la evolución por adaptación al medio, puede afirmar que su propuesta acepta que esas heurís-

${ }^{10}$ Esta crítica ha sido dirigida contra la propuesta de la racionalidad ecológica por Sergio Martínez, quien muestra cómo la idea de ecología que subyace en el proyecto $\mathrm{ABC}$ lleva implícita una relación simplificada entre agente y entorno, pues supone que el entorno está básicamente bien definido y estructurado y que es sólo al agente a quien se le otorga un papel activo. Así, es el agente quien va y toma del ambiente ciertas estructuras informacionales que se encuentran ahí y que en buena medida son invariables (Martínez 2009, p. 50).

${ }^{11} \mathrm{El}$ problema de cómo elegimos entre diferentes heurísticas es importante sobre todo en propuestas como la de Gigerenzer en las que el razonamiento heurístico se caracteriza como una lista de estrategias. Se trata de un problema que no ha sido resuelto en el caso de la racionalidad ecológica. El presente texto no recalca este problema, aunque sí otro afín, que es suponer que la relación entre el agente y su medio está de alguna manera clara y bien establecida, es decir, que va en una sola dirección. Esto es algo que de alguna manera está supuesto en la propuesta de la racionalidad ecológica y es parte de lo que se quiere señalar que debería cambiar para poder tener una ecología verdaderamente interactiva del razonamiento.

Revista de Filosofía Diánoia, vol. 64, no. 83 (noviembre de 2019-abril de 2020) e-ISSN: 1870-4913 • DOI: https://doi.org/10.22201/iifs.18704913e.2019.83.1618 
ticas son patrones estables de responder al ambiente y, por lo menos, afirmar que esos patrones se generaron en la interacción con el ambiente. Sin embargo, en su propuesta, la explicación de la constitución de las heurísticas tiene que ver más con la explicación de una adaptación biológica y se reconoce que es difícil tener la seguridad de que las heurísticas que modelan corresponden con las heurísticas que usamos los humanos (Todd 2001, p. 63). Pero, sobre todo, la explicación de cómo llegamos a adquirir las heurísticas, aunque deseable, es prescindible para dar cuenta de la racionalidad humana porque su propuesta puede plantearse sin ella.

Hay dos razones por las cuales sería importante contar con una ecología del razonamiento (de alguna manera están implícitas en el análisis previo sobre la racionalidad ecológica): una es poder elucidar lo que vimos que Simon llamaba "problemas mal estructurados"; la otra es explicar cómo generamos y modificamos a través de la experiencia nuestros patrones de decisión. La idea de los problemas mal estructurados es, en lo fundamental, que hay problemas que no son susceptibles de resolución en el computacionalismo clásico, en gran medida porque no es posible saber si tienen una respuesta correcta. Como Simon reconoce, se trata de la mayoría de los problemas que enfrentamos en nuestra vida cotidiana (Simon 1958, p. 5). Sin embargo, uno de los impedimentos para dar cuenta de este tipo de problemas es justo que no encajan con el tipo de explicación computacional que se quiere ofrecer para ellos. En el caso de la racionalidad ecológica de Gigerenzer, se conserva la idea de que las heurísticas rápidas y frugales deben ser susceptibles de interpretación en un modelo computacional y terminan por ser una conversión, como vimos, muy abstracta de esos problemas en problemas bien estructurados. Mi propuesta, dados los problemas que se presentan en la posición de Gigerenzer, es que vale la pena replantear la caracterización del razonamiento para estudiar ese tipo de problemas de una manera que no necesariamente requiera formular el razonamiento heurístico como una colección de algoritmos. Una de las implicaciones que se espera de este replanteamiento es poder dar una explicación de la generación y modificación de los patrones de razonamiento, más allá de contar con una explicación de las posibles condiciones ambientales que pudieron haber dado lugar a las heurísticas rápidas y frugales como producto de la adaptación por selección natural. El objetivo es motivar la idea de que este tipo de explicación es deseable y viable mediante la presentación de algunos casos empíricos que nos revelan un sentido más profundo en el que los estudios situados de la cognición pueden ofrecernos una mejor comprensión del razonamiento y la racionalidad,

Revista de Filosofía Diánoia, vol. 64, no. 83 (noviembre de 2019-abril de 2020) e-ISSN: 1870-4913 • DOI: https://doi.org/10.22201/iifs.18704913e.2019.83.1618 
a saber, entender la ecología del razonamiento considerando que las interacciones con el entorno son parte de las estructuras cognitivas que sustentan nuestras capacidades de razonamiento. Este replanteamiento no necesariamente implica el abandono de los modelos que formulan Gigerenzer y el grupo ABC, ni tampoco el satisfaccionismo de Simon; más bien se trata de complementar estas propuestas para los casos en que los problemas enfrentados no pueden convertirse en problemas bien estructurados. Una pretensión que quizá debe abandonarse es considerar que esos modelos son representaciones adecuadas de los procesos cognitivos que de hecho tienen lugar cuando razonamos.

En esta sección presento algunos aspectos complementarios que deberían tomarse en cuenta para entender la ecología del razonamiento de una forma interactiva, es decir, de manera que el ambiente se considere parte relevante de la caracterización del proceso de razonamiento y se traten de explicar las transformaciones de nuestros procesos de razonamiento heurístico. Lo que me interesa subrayar es que una parte importante de entender la racionalidad humana y el razonamiento heurístico es dar cuenta de cómo las circunstancias de nuestra corporalidad, nuestro entorno social y material pueden restringir, dar forma y alterar el uso de las estrategias cognitivas. Esta comprensión debe incluir una consideración de los agentes como seres activos en la estructuración de sus ambientes y actividades con miras a lograr un objetivo; no basta con caracterizar agentes que se enfocan en el reconocimiento de los principios inferenciales que les permiten resolver acertadamente (o con cierta razonabilidad) un problema con características estructurales específicas de la información.

Como veremos a continuación con algunos estudios empíricos, tanto recientes como algunos que se han vuelto clásicos, la modalidad en la que se presenta la información y el contexto material y social donde se enfrenta el problema -incluso tratándose de problemas bien estructurados - son relevantes para el proceso de solución que los agentes siguen. Ambas ideas parecen tener relevancia en la formulación de la racionalidad ecológica de $\mathrm{ABC}$ desde sus inicios; ${ }^{12}$ sin embargo,

${ }^{12}$ Quizá incluso aparecen de manera más interesante en sus inicios. Por ejemplo, la idea de que el contexto social importa en la toma de decisiones aparece muy claro en "Rationality. Why Social Context Matters" (Gigerenzer 1996), donde se argumenta justamente que no es posible hablar de racionalidad a partir de ciertas reglas de inferencia sin considerar el contexto social (y eso incluye los objetivos, las motivaciones y los valores del agente) en el análisis de la decisión. Pero no está claro cómo se puede insertar después este tipo de consideraciones bajo la idea más específica de que cada heurística tiene un ambiente (estructura informacional)

Revista de Filosofía Diánoia, vol. 64, no. 83 (noviembre de 2019-abril de 2020) e-ISSN: 1870-4913 • DOI: https://doi.org/10.22201/iifs.18704913e.2019.83.1618 
como hemos visto, la manera de entender, por un lado, la normatividad de las heurísticas como el aparejamiento apropiado de las estrategias de decisión con la estructura informacional del ambiente y, por otro, el tipo de relación que esto supone entre el agente y su medio dan muy poca importancia a la corporeización y al modo en que el agente percibe la información relevante para la solución de la tarea. La modalidad en que se presenta la información es relevante en el proyecto de la racionalidad ecológica porque permite identificar si cierto tipo de estrategias serán consideradas normativas o no, dado que su formato representacional es fácil de procesar por los humanos $\mathrm{y}$, por lo tanto, se pueden aplicar adecuadamente. ${ }^{13}$ De manera que, aunque el formato representacional de la información tiene gran importancia para el aspecto normativo de la propuesta, parece no considerarse del todo en su manera de caracterizar las heurísticas del razonamiento. Esto, que considero que podemos ver a través del análisis hasta ahora presentado, se debe a la manera en que se caracteriza la cognición a través de una distinción tajante entre funciones cognitivas superiores e inferiores y tomando en consideración sólo las superiores.

La referencia que haré a ciertos estudios empíricos tiene el propósito de ejemplificar cómo el razonamiento simbólico se basa en aquello que percibimos en un sentido que va más allá de ser un mero insumo para la aplicación de una regla. La intención es señalar que el razonamiento heurístico está bastante más corporeizado y atrincherado en nuestro entorno social y material de lo que Gigerenzer y sus colegas suponen. Los primeros estudios muestran que la resolución de problemas y la toma de decisiones es algo que va más allá de reconocer sólo ciertos rasgos del ambiente para aplicar una regla. Los segundos muestran que el modo en el cual se presentan los problemas, incluso los problemas matemáticos — bien estructurados-, tiene repercusiones en la manera de resolverlos (Landy y Goldstone 2007; Landy, Allen y Zednik 2014). Los terceros son ejemplos de la descarga cognitiva que hacemos sobre el ambiente material y de cómo modificamos —a través de esa descarga

con el cual resulta exitosa, de manera que parte de la conducta ecológicamente racional es reconocer esas estructuras informacionales y emplear la heurística que en ese caso sería exitosa.

${ }^{13}$ Un ejemplo de ello es la afirmación de Gigerenzer 1996 de que no podemos formular un juicio negativo respecto de la solución de problemas probabilísticos cuando la información se presenta en términos de probabilidad subjetiva y no en términos frecuentistas, debido a que no somos proclives a entender las probabilidades subjetivas y sí las frecuencias. Ésta es la parte central de la discusión con Kahneman y la tradición de heurística y sesgo. Véase Fonseca Patrón 2016.

Revista de Filosofía Diánoia, vol. 64, no. 83 (noviembre de 2019-abril de 2020) e-ISSN: 1870-4913 • DOI: https://doi.org/10.22201/iifs.18704913e.2019.83.1618 
en el medio material- nuestras estrategias de resolución del mismo problema a través del tiempo. Como veremos, hay razones para pensar que, a lo largo de nuestra vida, cambiamos nuestras estrategias para resolver incluso el mismo tipo de problemas dadas nuestras capacidades cognitivas (intelectuales, perceptuales y motoras) y nuestra relación con el entorno social y material.

El supuesto de que las respuestas observadas en pruebas de laboratorio permiten hacer una buena inferencia de las capacidades cognitivas que empleamos en problemas estructuralmente similares en la vida cotidiana está bastante extendido en la psicología del razonamiento. Es un supuesto que podemos encontrar en la propuesta de la racionalidad ecológica, pues las caracterizaciones de los ambientes en los cuales las heurísticas rápidas y frugales son exitosos (o aquellos) en los que se prueba su éxito mediante simulaciones computacionales son descripciones abstractas y muy bien definidas de los problemas, como las que se utilizan en las pruebas de laboratorio. $\mathrm{Y}$ es a partir de estas características de los ambientes y problemas que se infieren las heurísticas cognitivas. Sin embargo, este supuesto se puede cuestionar con base en observaciones empíricas. Jane Lave, por ejemplo, presenta estudios que analizan las prácticas aritméticas en diferentes grupos poblacionales. Uno de esos grupos está formado por niños que estudian por la mañana y que por las tardes venden diversos productos en un mercado en Brasil. Los niños fueron abordados en el mercado por los entrevistadores quienes se presentaron como clientes. En el caso de un niño de 12 años que vendía cocos, el entrevistador-cliente le preguntó cuánto costaba un coco, a lo que el niño contestó que 35 reales. El entrevistador-cliente le dijo entonces que quería 10 cocos y le preguntó cuánto era. El niño se quedó pensando e hizo el siguiente procedimiento para determinar el monto total: "Tres serían 105, con tres más serían 210. (Pausa) Necesito cuatro más. Esto es. . (pausa) 315. . Creo que son 350" (Carraher, Carraher y Schliemann 1982; Carraher y Schliemann 1982, cit. en Lave 1988, pp. 65). Aunque el niño cursaba el tercer grado de la escuela básica en el cual les enseñan a multiplicar por 10 agregando sólo un cero, el niño optó por resolver el problema representándolo en otra forma.

La conclusión que le interesa mostrar a Lave con este ejemplo y otros que presenta es que no existe una transferencia directa del conocimiento teórico enseñado en las aulas a las situaciones de la vida cotidiana en las que, en principio, se podrían usar esos conocimientos. Lo que explica que no se pueda transferir el conocimiento proporcionado en el salón de clase a las situaciones reales es la falta del contexto en el que se presentan los problemas. Lo que concluye Lave sobre la activi-

Revista de Filosofía Diánoia, vol. 64, no. 83 (noviembre de 2019-abril de 2020) e-ISSN: 1870-4913 • DOI: https://doi.org/10.22201/iifs.18704913e.2019.83.1618 
dad aritmética del ejemplo es que las actividades que realizamos están constituidas en formas específicas de acuerdo con la situación y que son esas relaciones entre actividades y situaciones las que dan forma a nuestra práctica cognitiva. Un señalamiento importante es, entonces, la poca confianza que podemos tener en que, si una persona conoce cierta regla de inferencia (matemática o lógica) de forma teórica, la va a aplicar en casos concretos de su vida. En este sentido, un proyecto que caracteriza el razonamiento heurístico como una lista de algoritmos que son adecuados en contextos particulares, pero no da cuenta de la forma en que esos contextos afectan la toma de decisiones es incompleto.

Landy, Allen y Zednik 2014 presentan una imagen del razonamiento abstracto como un tipo especial de razonamiento corporeizado y hacen énfasis en mostrar cómo el razonamiento simbólico se aplica comúnmente mediante un involucramiento perceptivo motor con estructuras ambientales concretas. Antes de llegar a esta imagen del razonamiento abstracto como corporeizado Landy y Goldstone 2007 llevaron a cabo experimentos en los que exploraron el papel del acomodo visual en juicios que debían realizarse siguiendo reglas sintácticas. Para ello pidieron a los participantes que juzgaran si algunas ecuaciones algebraicas simples eran válidas, es decir, si las expresiones a ambos lados de la ecuación eran equivalentes como en: $5+x=6+x-1$. Se les presentaron tanto ecuaciones válidas como inválidas y esa validez podía ser sensible o no al orden adecuado de las operaciones. Es decir, se agruparon visualmente las operaciones de las ecuaciones de la siguiente manera:

a) consistente: que coincidieran con la manera correcta de agrupar formalmente las operaciones; como en: $h+q * t+n=h+t * q+n$.

b) inconsistente: que no coincidieran con la manera correcta de agrupar formalmente las operaciones; como en: $t+p * m+f=$ $t+p * m+f$.

c) neutral: que no hubiera un agrupamiento particular; como en: $t+j * n+e=n+e * t+j$.

Cada una de las variaciones matemáticas se presentó en cada una de esas tres representaciones. La hipótesis era que, si el razonamiento algebraico emplea el agrupamiento visual como grupos formales, entonces el agrupamiento inducido por las diferencias espaciales debería influenciar en forma sistemática el orden de las operaciones empleadas en la tarea de validación.

Revista de Filosofía Diánoia, vol. 64, no. 83 (noviembre de 2019-abril de 2020) e-ISSN: 1870-4913 • DOI: https://doi.org/10.22201/iifs.18704913e.2019.83.1618 
La conclusión fue que el anclaje físico es importante incluso cuando los agentes procesan notaciones simbólicas y, más en general, que el razonamiento formal es más visual de lo que usualmente se reconoce. En el caso particular del presente artículo, esos experimentos son importantes porque muestran que nuestro razonamiento va más allá de elegir una regla una vez que se ha reconocido ciertas características informacionales y que nuestra corporalidad desempeña un papel más complejo que sólo proveer los insumos para la aplicación de una heurística.

Por último, otro tipo de estudios que nos revelan la importancia de la corporalidad y el entorno social son Salthouse 1987, Salthouse 2012, Kristiansson 2013 y Dahlbäck, Kristiansson y Stjernberg 2013. Salthouse ha trabajado durante varias décadas en cómo explicar las discrepancias encontradas entre "por un lado, las competencias para adultos mayores, dadas por sentadas sobre la base de las observaciones de la vida diaria y, por otro lado, sus competencias inferidas a partir de resultados de laboratorio" (Salthouse 1987, cit. en Salthouse 2012, p. 202). Este autor ha señalado que tales discrepancias pueden interpretarse, entre otras maneras, como un indicador de que los procesos que los agentes emplean en la vida cotidiana son distintos de los procesos cognitivos empleados en el laboratorio, o al menos no se restringen a ellos (Salthouse 2012) ${ }^{14}$ y, sobre todo, que pueden variar a lo largo de la vida del agente en función tanto del estado de las capacidades cognitivas como de los recursos materiales y sociales disponibles. Este tipo de estudios socava la idea de que los procesos cognitivos que se infieren con la realización de pruebas de laboratorio — que aíslan los problemas de sus contextos tanto materiales como sociales - nos permiten afir-

\footnotetext{
${ }^{14}$ Salthouse menciona cuatro posibles explicaciones del hecho de que no haya mayores consecuencias en la vida cotidiana por el declive cognitivo relacionado con el envejecimiento reportado por las pruebas de laboratorio: 1) que las actividades de la vida cotidiana no requieran ejecutar al máximo las capacidades cognitivas; 2) que las habilidades evaluadas en las pruebas experimentales determinan el nivel de ejecución cuando uno no sabe qué hacer y no tiene información relevante, mientras que, por lo general, en la vida cotidiana sólo es ligeramente diferente a cosas que hemos realizado en el pasado; 3) considerar que la ejecución no es lo único que determina el éxito de las actividades cotidianas, y 4) que las personas suelen hacer arreglos especiales para compensar las actividades que les van costando cada vez más trabajo, como evitar realizar ciertas actividades (manejar, por ejemplo), delegar responsabilidades en terceras personas o adaptar su espacio para desempeñar en forma adecuada las misma actividades que antes. Salthouse no pretende en su artículo elucidar cuál de esas posibles explicaciones es la correcta, sino señalar que las discrepancias más bien pueden deberse a una combinación de diversos factores, como los cuatro mencionados.
}

Revista de Filosofía Diánoia, vol. 64, no. 83 (noviembre de 2019-abril de 2020) e-ISSN: 1870-4913 • DOI: https://doi.org/10.22201/iifs.18704913e.2019.83.1618 
mar con precisión que los procesos cognitivos que de hecho se emplean en situaciones de la vida cotidiana son aquellos que cumplen con la estructura evaluada en los problemas del laboratorio o, por lo menos, que eso varía en diferentes momentos de nuestra vida. ${ }^{15}$ Los estudios de Lave pueden indicarnos cómo incluso en casos de problemas bien estructurados, como los aritméticos, llegar a la solución va más allá de sólo reconocer ciertos rasgos del ambiente y aplicar una regla de inferencia ya conocida. Los estudios de Salthouse pueden servirnos para indicar eso mismo en problemas mal estructurados. Así, una implicación de cuestionar el supuesto de que podemos inferir claramente los procesos cognitivos a partir de pruebas de laboratorio y simulaciones computacionales es que, si lo que queremos entender es cómo resolvemos problemas mal estructurados, tendríamos que explicar la forma en que el agente y su medio se influyen mutuamente y posibilitan la generación, estabilización y modificación de patrones de acción. Los estudios de Landy sobre la importancia de la modalidad también pueden considerarse un refuerzo de esta afirmación.

En conformidad con la línea de estudios sobre el declive de las capacidades cognitivas a través del proceso de envejecimiento, Kristiansson 2013 y Dahlbäck, Kristiansson y Stjernberg 2013 presentan estudios que revelan cómo las personas al envejecer son capaces de llevar a cabo ciertas tareas estructuralmente similares a las que emprenden bajo condiciones de laboratorio de forma óptima en su vida cotidiana - aun después de percibirse un declive de ciertas capacidades cognitivas-. De esto los autores concluyen que la ejecución de las tareas de la vida cotidiana que requieren capacidades cognitivas que ya están en declive se realiza recurriendo al entorno. La idea que me interesa resaltar de estos estudios es la afirmación de que la ejecución de diversas estrategias cognitivas se sustenta en la interacción del agente con su entorno; en particular, en la manipulación y en la construcción de su entorno social y material. Si esto es así, entonces no parece adecuado considerar que podemos explicar el razonamiento heurístico, o por lo menos no completamente, o no en todos los casos, a partir de un conjunto de estrategias inferenciales bien definidas que se pueden aplicar en diversos contextos materiales y sociales por igual. Aquí también se vuelve importante subrayar cómo en estos casos el ambiente ya no puede ca-

\footnotetext{
${ }^{15}$ Eso tiene que ver con el supuesto de Simon de delinear el razonamiento como el razonamiento de un humano con características muy específicas: "adulto inteligente en nuestra propia cultura" (Newell y Simon 1972, p. 2, cit. en HendriksJansen 1996, p. 21). Esto no permite entender los procesos de obtención y modificación de los procesos cognitivos.
}

Revista de Filosofía Diánoia, vol. 64, no. 83 (noviembre de 2019-abril de 2020) e-ISSN: 1870-4913 • DOI: https://doi.org/10.22201/iifs.18704913e.2019.83.1618 
racterizarse sólo como la estructura informacional que empata en forma adecuada con la estructura inferencial de una heurística rápida y frugal.

El ambiente en los casos estudiados por Lave, Landy y sus colegas, Salthouse, Kristiansson y Dahlbäck y sus colegas desempeña un papel activo en el proceso mediante el cual se lleva a cabo alguna tarea; los agentes no sólo buscan en el ambiente patrones de información para aplicar una regla de inferencia, sino que usan y transforman diferentes rasgos del ambiente a su alcance para lograr sus objetivos. ${ }^{16}$ En estos casos, la unidad de análisis de los procesos cognitivos es la persona en acción, incluido el ambiente físico y social en el que se lleva a cabo la actividad. Tomar en cuenta este papel activo tanto del agente como del ambiente requiere entender la ecología del razonamiento de una forma interactiva.

Entender la ecología del razonamiento en una forma interactiva en el sentido sugerido permitiría dar cuenta, por un lado, de cómo los agentes solemos emplear el ambiente como parte de nuestras capacidades de razonamiento y, por otra, de cómo suceden las modificaciones de los patrones de razonamiento heurístico a través del tiempo. Un propósito central de este artículo es hacer ver que vale la pena revisar la caracterización que por lo común se hace del razonamiento heurístico y de la metodología que, por lo tanto, resulta adecuada para estudiarlo. Una manera en que se puede complementar la caracterización del razonamiento heurístico de Gigerenzer es entenderlo como una capacidad que permite generar formas particulares de acción, patrones o modos estables de responder al ambiente que son producto de la interacción social y de la interacción de los agentes con su ambiente. Esto permite comenzar a analizar cómo se adquieren esos patrones a través de la socialización y el aprendizaje, cómo dependen del contexto y no

\footnotetext{
${ }^{16}$ Algunos estudios, como Bardone 2011 o Martínez 2016, recuperan la noción de "nicho ecológico" para analizar esa interactividad de la ecología del razonamiento. En la teoría de los nichos, el ambiente se considera una fuente de recursos para los organismos que los habitan y son los organismos quienes tratan de modificar ese entorno para explotar los elementos que en él se encuentran. Bardone habla de "nichos cognitivos" para referirse a las formas en que los agentes modifican el ambiente para extender sus capacidades cognitivas o mitigar sus limitaciones; en este caso, la interacción con el ambiente se entiende como un proceso de modificaciones que pueden acumularse y, así, llevar a su vez a modificaciones cognitivas (Bardone 2011, p. 60). Además considera que es esta posibilidad de modificación continua entre el agente y el entorno lo que permite desarrollar mejores estrategias de decisión, pero argumenta que esta interacción no se encuentra en la propuesta ecológica del grupo ABC (Bardone 2011, pp. 35-39). Sin embargo, aún se discute cómo debe caracterizarse exactamente esa interacción.
}

Revista de Filosofía Diánoia, vol. 64, no. 83 (noviembre de 2019-abril de 2020) e-ISSN: 1870-4913 • DOI: https://doi.org/10.22201/iifs.18704913e.2019.83.1618 
sólo apelar a que son producto de una adaptación al medio durante el Pleistoceno. Pero hay aún otra implicación, a saber, si entendemos la ecología del razonamiento en un sentido interactivo, como el sugerido aquí, tenemos una pista respecto de cómo reconfigurar la imagen de la racionalidad humana a partir de una caracterización situada de la cognición. Una afirmación cada vez más común es que la racionalidad ha de configurarse a partir de la comprensión de los patrones estables de interacción entre ambientes y agentes (Clark 2001, Martínez 2009, Bardone 2011); considero que el análisis presentado nos permite reconocer que ese objetivo se puede lograr mediante la comprensión de la ecología del razonamiento. Al entender el razonamiento como patrones de acción, surge también la posibilidad de evaluar esos patrones en términos de si nos permiten o no lograr cierto objetivo. Y ese objetivo no tiene por qué considerarse necesariamente la única respuesta posible de un problema, es decir, de esta manera podemos evaluar los patrones de razonamiento incluso en casos de problemas mal estructurados.

Si este análisis es correcto, la tarea siguiente será estudiar el razonamiento a través de la observación de los patrones de acción mediante los cuales respondemos ante el ambiente considerando que el papel de la interacción social y de la interacción de los agentes con su ambiente material es crucial para la generación, estabilización y modificación de esos patrones. Una explicación de este tipo no excluye la posibilidad de que a la larga sea posible formular modelos computacionales de ellos; se trata de no suponer por anticipado que nuestros patrones de razonamiento deben ser susceptibles de un análisis computacional. Este tipo de explicación tampoco excluye la idea de que nuestra historia evolutiva desempeñe un papel importante, pero sí supone aceptar que la explicación evolutiva es bastante más compleja que la adaptación al medio por selección natural en el Pleistoceno y que, de alguna manera, esa historia incluye la interacción social y material de los agentes.

\section{Conclusión}

A partir del análisis de los estudios del grupo $\mathrm{ABC}$ podemos concluir dos cosas: 1) que si bien la propuesta de racionalidad ecológica es un intento por entender de forma situada la racionalidad humana, no se trata de una propuesta que sitúe el razonamiento, y 2) una manera de complementar esa propuesta es considerar en mayor medida la importancia que tiene la corporalidad de los agentes y su interacción con su entorno social y material en la elección y modificación de los procesos de razonamiento heurístico.

Revista de Filosofía Diánoia, vol. 64, no. 83 (noviembre de 2019-abril de 2020) e-ISSN: 1870-4913 • DOI: https://doi.org/10.22201/iifs.18704913e.2019.83.1618 
La racionalidad ecológica de Gigerenzer incorpora de alguna manera el ambiente al otorgarle un papel importante en la normatividad, pero la caracterización del ambiente se reduce a ciertas estructuras informacionales a las cuales las heurísticas deben acoplarse de forma adecuada. Si bien puede concebirse como situada, esta imagen de la racionalidad no parece incluir una ecología del razonamiento. Se trata de una propuesta de racionalidad ecológica, pero no desarrolla propiamente una ecología del razonamiento. En particular, requiere la transformación de los problemas de la vida cotidiana, que podemos asociar con lo que Simon llamaba problemas mal estructurados, en problemas bien estructurados; dicha transformación permite modelar la toma de decisiones y la solución de problemas a través de algoritmos (las heurísticas rápidas y frugales). Sin embargo, esto implica que tener una conducta racional es algo que se evalúa sólo a través de cortes de tiempo discretos, lo único que se toma en cuenta el momento en que se toma la decisión. Sobre todo, implica que, al igual que en la estándar de la racionalidad, la conducta racional se refiere a la identificación de una regla de inferencia y su aplicación correcta. Esta manera de caracterizar el razonamiento heurístico dificulta entender cómo se modifica nuestro razonamiento para encontrar nuevas maneras de resolver problemas; en este sentido, también es una visión estática de la ecología del razonamiento.

A partir de ese análisis se concluye que una manera de complementar la racionalidad ecológica es a través del desarrollo de una ecología interactiva del razonamiento. Se trataría de una ecología del razonamiento que tome en cuenta la corporalidad del agente, su interacción con el medio social y material como parte relevante del proceso de razonamiento. Esta afirmación se respalda en diversos estudios empíricos que muestran desde diversas perspectivas cómo la modalidad en la que se presenta la información afecta nuestra percepción de los problemas y la manera en que intentamos resolverlos, lo que nos da una pista de la importancia de la corporeización, que va más allá de proveernos ciertos insumos para la aplicación de determinados algoritmos. Los estudios también muestran cómo las soluciones que ofrecemos a los problemas varían en función de los recursos ambientales (tanto sociales como materiales) que tenemos a nuestro alcance. Por ello, la sugerencia es complementar las heurísticas rápidas y frugales con una caracterización del razonamiento heurístico como patrones estables para responder al ambiente que se generan a través de la interacción social y de la interacción del agente con su medio. Entender los patrones estables de interacción del agente y su medio facilitaría explicar cómo modifica-

Revista de Filosofía Diánoia, vol. 64, no. 83 (noviembre de 2019-abril de 2020) e-ISSN: 1870-4913 • DOI: https://doi.org/10.22201/iifs.18704913e.2019.83.1618 
mos nuestro razonamiento a lo largo del tiempo; en particular, entender cómo el razonamiento heurístico participa en nuestra conformación de una imagen del mundo que, en circunstancias específicas, nos permite responder de manera creativa a situaciones similares, pero no necesariamente idénticas en términos estructurales, a aquellas que hemos observado antes. ${ }^{17}$

\section{BIBLIOGRAFÍA}

Bardone, E., 2011, Seeking Chances. From Biased Rationality to Distributed Cognition, Springer, Berlín.

Brighton, H. y P.M. Todd, 2009, "Situating Rationality. Ecologically Rational Decision Making with Simple Heuristics", en Robbins y Aydede 2009b, pp. 322-346.

Brunswik, E., 1955, "Representative Design and Probabilistic Theory in a Functional Psychology", Psychological Review, vol. 62, no. 3, pp. 193-217.

Carraher, T.N., D.W. Carraher y A.D. Schliemann, 1982, "Na vida dez; na escola, zero: os contextos culturais da aprendizagem da matemática", Cadernos de Pesquisa, vol. 42, pp. 79-86.

Carraher, T.N. y A.D. Schliemann, 1982, "Computation Routines Prescribed by Schools: Help or Hindrance?", artículo presentado en la Conferencia de la OTAN para la Adquisición de Habilidades Simbólicas y publicado en 1985 en Journal for Research in Mathematics Education, vol. 16, no. 1, pp. 37-44.

Clancey, W.J., 2009, "Scientific Antecedents of Situated Cognition", en Robbins y Aydede 2009b, pp. 11-34.

Clark, A., 2001, "Reason, Robots and the Extended Mind (Rationality for the New Millenium)", Mind and Language, vol. 16, no. 2, pp. 121-145.

Clark, A. y D. Chalmers, 1998, "The Extended Mind", Analysis, vol. 58, no. 1, pp. 7-19.

Dahlbäck, N., M. Kristiansson y F. Stjernberg, 2013, "Distributed Remembering through Active Structuring of Activities and Environments", Review of Philosophy and Psychology, vol. 4, no. 1, pp. 153-165.

Fonseca Patrón, A.L., 2016, "El debate sobre las heurísticas. Una disputa sobre los criterios de buen razonamiento entre la Tradición de Heurística y Sesgo y la Racionalidad Ecológica", Valenciana, vol. 9, no. 17, pp. 87-115.

Gigerenzer, G., 1996, "Rationality: Why Social Context Matters", en P.B. Baltes y U. Staudinger (comps.), Interactive Minds, Cambridge University Press, Nueva York, pp. 319-346.

_, 2001 , "The Adaptive Toolbox", en G. Gigerenzer y R. Selten (comps.), Bounded Rationality. The Adaptive Toolbox, The MIT Press, Londres, pp. 3750 .

${ }^{17}$ La autora agradece al proyecto PAPIIT IN 402018 "Cognición, artefactualidad y representación en la ciencia” por haber apoyado la realización de este artículo. 
Gigerenzer, G. y H. Brighton, 2009, "Homo Heureticus: Why Biased Minds Make Better Inferences", Topics in Cognitive Science, vol. 1, no. 1, pp. 107143.

Gigerenzer, G. y D.G. Goldstein, 1999, "Betting on One Good Reason. The Take the Best Heuristic", en G. Gigerenzer, P.M. Todd y grupo de investigación ABC, Simple Heuristics That Make Us Smart, Oxford University Press, Nueva York, pp. 75-96.

Gigerenzer, G. y T. Sturm, 2012, "How (Far) Can Rationality Be Naturalized?", Synthese, vol. 187, no. 1, pp. 243-268.

Gigerenzer, G. y P.M. Todd, 1999, "Fast and Frugal Heuristics. The Adaptive Toolbox", en G. Gigerenzer, P.M. Todd y grupo de investigación ABC, Simple Heuristics That Make Us Smart, Oxford University Press, Nueva York, pp. 335.

Hendriks-Jansen, H., 1996, Catching Ourselves in the Act. Situated Activity, Interactive Emergence, Evolution, and Human Thought, The MIT Press, Cambridge, Mass.

Hutchins, E., 2001, "Distributed Cognition", en N.J. Smelser y P.B. Baltes (comps.), The International Encyclopedia of the Social and Behavioral Sciences, Elsevier, Ámsterdam, pp. 2068-2072.

— 2010, "Cognitive Ecology", Topics in Cognitive Science, vol. 2, no. 4, pp. 705-715.

Kirsh, D. y Maglio, P., 1994, "On Distinguishing Epistemic from Pragmatic Action", Cognitive Science, vol. 18, pp. 513-549.

Kristiansson, M., 2013, "The Case of Cognitive Ecology for Cognitive Processes in Everyday Life Situations", en M. Knauff, M. Pauen, N. Sebanz e I. Wachsmuth (comps.), Cognitive Minds: Social Interaction and Group Dynamics. Proceedings of the 35th Annual Conference of the Cognitive Science Society, Cognitive Science Society, Berlín, pp. 2778-2783.

Landy, D., C. Allen y C. Zednik, 2014, "A Perceptual Account of Symbolic Reasoning", Frontiers in Psychology, vol. 5, pp. 1-10.

Landy, D. y R.L. Goldstone, 2007, "How Abstract Is Symbolic Thought?", Journal of Experimental Psychology. Learning, Memory and Cognition, vol. 33, no. 4, pp. 720-733.

Lave, J., 1988, Cognition in Practice. Mind, Mathematics and Culture in Everyday Life, Cambridge University Press, Cambridge.

Marsh, B., P.M. Todd y G. Gigerenzer, 2004, "Cognitive Heuristics. Reasoning the Fast and Frugal Way", en J.P. Leighton y R.J. Sternberg (comps.), The Nature of Reasoning, Cambridge University Press, Nueva York, pp. 273-287.

Martínez, S.F., 2009, "Hacia una racionalidad ecológica distribuida en prácticas", en A. Eraña y G. Mateos (comps.), La cognición como proceso cultural, Centro de Estudios Multidisciplinarios-UnAm, México, pp. 21-53.

__ 2016, "Cultura material y cognición social”, en P. Hernández, J. García y M. Romo (comps.), Cognición. Estudios multidisciplinarios, Centro de Estudios Filosóficos, Políticos y Sociales Vicente Lombardo Toledano, México, pp. 247-264.

Revista de Filosofía Diánoia, vol. 64, no. 83 (noviembre de 2019-abril de 2020) e-ISSN: 1870-4913 • DOI: https://doi.org/10.22201/iifs.18704913e.2019.83.1618 
Newell, A. y H.A. Simon, 1976, "Computer Science as Empirical Inquiry. Symbols and Search", Communications of the ACM, vol. 19, no. 3, pp. 113-126.

Robbins, P. y M. Aydede, 2009a, "A Short Primer on Situated Cognition", en Robbins y Aydede 2009b, pp. 3-10.

Robbins, P. y M. Aydede (comps.), 2009b, The Cambridge Handbook of Situated Cognition, Cambridge University Press, Nueva York.

Salthouse, T., 1987, "Age, Experience, and Compensation", en C. Schooler y K.W. Schaie (comps.), Cognitive Functioning and Social Structures over the Life Course, Ablex, Norwood, Nueva Jersey, pp. 142-57.

- , 2012, "Consequences of Age-Related Cognitive Declines", Annual Review of Psychology, vol. 63, pp. 201-26.

Shapiro, L., 2011, Embodied Cognition, Routledge, Nueva York.

Shepard, R.N., 2001, "Perceptual-Cognitive Universals as Reflections of the World", Behavioral and Brain Sciences, vol. 24, no. 4, pp. 581-601.

Simon, H.A., 1958, "Heuristic Problem Solving: The Next Advance in Operations Research", Operations Research, vol. 6, no. 1, pp. 1-10.

— 1981 , The Sciences of the Artificial, 2a. ed., The MIT Press, Cambridge, Mass.

— , 1990, "Invariants of Human Behavior", Annual Review of Psychology, vol. 41, pp. 1-19.

Smith, B.C., 1999, "Situatedness/Embeddedness", en R.A. Wilson y F.C. Keil (comps.), The MIT Encyclopedia of The Cognitive Sciences, The MIT Press, Cambridge, Mass., pp. 769-770.

Stein, E., 1996, Without Good Reason. The Rationality Debate in Philosophy and Cognitive Science, Clarendon Press, Oxford.

Todd, P.M., 2001, "Fast and Frugal Heuristics for Environmentally Bounded Minds", en G. Gigerenzer y R. Selten (comps.), Bounded Rationality. The Adaptive Toolbox, The MIT Press, Cambridge, Mass. pp. 51-70.

Vera, A.H. y H.A. Simon, 1993a, "Situated Action. A Symbolic Interpretation", Cognitive Science, no. 17, vol. 1, pp. 7-48.

—, 1993 b, "Situated Action. Reply to Reviewers", Cognitive Science, no. 17, no. 1, pp. 77-86.

Recibido el 2 de junio de 2018; revisado el 8 de marzo de 2019; aceptado el 12 de abril de 2019. 